Revue trimestrielle sur l'image géographique et les formes du territoire

$131 \mid 2021$

Varia

\title{
Aux deux extrémités d'une route transnationale : spatialiser les marchés transnationaux entre l'Algérie et la Chine
}

At both ends of a transnational route: spatializing trading posts between Algeria and China

Los dos extremos de una ruta transnacional: espacialización de los puntos comerciales entre Argelia y China

\section{Saïd Belguidoum et Olivier Pliez}

\section{(2) OpenEdition}

\section{Journals}

Édition électronique

URL : https://journals.openedition.org/mappemonde/6143

DOI : $10.4000 /$ mappemonde.6143

ISSN : 1769-7298

\section{Éditeur}

UMR ESPACE

Référence électronique

Saïd Belguidoum et Olivier Pliez, « Aux deux extrémités d'une route transnationale : spatialiser les marchés transnationaux entre l'Algérie et la Chine », Mappemonde [En ligne], 131 | 2021, mis en ligne le 08 juillet 2021, consulté le 14 juillet 2021. URL : http://journals.openedition.org/mappemonde/6143 DOI : https://doi.org/10.4000/mappemonde.6143

Ce document a été généré automatiquement le 14 juillet 2021. 


\title{
Aux deux extrémités d'une route transnationale : spatialiser les marchés transnationaux entre l'Algérie et la Chine
}

\author{
At both ends of a transnational route: spatializing trading posts between Algeria \\ and China
}

Los dos extremos de una ruta transnacional: espacialización de los puntos comerciales entre Argelia y China

Saïd Belguidoum et Olivier Pliez

\section{Introduction}

1 Si les travaux de recherche sur les réseaux commerciaux transnationaux qui relient le Monde arabe à la Chine se sont multipliés depuis une vingtaine d'années ${ }^{1}$, on constate que l'intérêt des chercheurs porte encore souvent sur les modalités selon lesquelles se créent des liens sociaux et/ou spatiaux entre des places marchandes aussi distantes et comment ces liens évoluent dans le temps. De nombreuses recherches ont ainsi été menées sur des places marchandes situées partout dans le monde, au Nord comme au Sud, des plus emblématiques aux plus discrètes, formant autant de maillons des chaînes d'approvisionnement qui traversent les continents. Ces travaux s'attachent ainsi à définir le caractère transnational, plutôt qu'international, des marchés choisis et collectent des matériaux empiriques privilégiant les descriptions qualitatives, en combinant les outils de la géographie urbaine (relevés, cartographie, collecte d'indicateurs) et de la sociologie compréhensive (entretiens, observation) afin de mettre en lumière de manière située les articulations entre un réseau spatial et un réseau social. 
2 Pour comprendre ce choix, plusieurs raisons peuvent être avancées. Ces travaux relèvent du champ du transnational et plus précisément de ce que l'on place sous l'expression générique de la mondialisation par le bas, balisée dès la fin des années 1980 aux États-Unis (Portes, 1997) et en Europe (Tarrius, 2002). Cette forme particulière de la mondialisation, parfois qualifiée de "capitalisme des parias $»^{2}$, économiquement modeste, mais socialement intense, demeure difficile à appréhender, car elle « englobe les opérations à petite échelle, l'économie informelle, les réseaux ethniques et diasporiques et les migrants internationaux $»^{3}$. Lorsque les innombrables microinteractions que ces flux génèrent atteignent le niveau de ce qu'Alain Tarrius (2002), un pionnier du champ, qualifie de « haute densité relationnelle », un comptoir est identifié et son ethnographie devient possible, mais pour autant pas sa mesure.

3 Le comptoir se caractérise par sa propension à générer des relations parce qu'il occupe une position centrale à la croisée de plusieurs routes. Or celles-ci sont plus difficiles à appréhender puisque l'on n'en connaît a priori ni la longueur, ni les ramifications, ni l'intégralité des espaces qui les jalonnent; or si les comptoirs (Bertoncello et al., 2009) sont les plus connus et les plus aisés à repérer, ils n'en demeurent pas moins qu'une forme parmi d'autres, aux côtés de multiples et anonymes lieux de transbordement, de stockage, de passage du gros au demi-gros puis au détail... De telles investigations ont connu un réel engouement depuis deux décennies. De nombreux chercheurs se sont en effet lancés dans la reconstitution de la route de tel ou tel autre produit circulant à travers la planète (Choplin, Pliez, 2018) tels que les papayes (Cook et al., 2004), les teeshirts (Rivoli, 2007), les sandales (Knowles, 2014), etc. En revanche, pour les institutions internationales, ces routes sont souvent encore amalgamées à celles des activités illicites et notamment celles des marchandises contrefaites (OECD/EUIPO, 2017). Porter le regard sur la seule contrefaçon offre l'avantage de pouvoir circonscrire des indicateurs mesurables - notamment celui de la valeur financière des marchandises saisies - et ainsi d'évaluer le poids économique des voies d'échange, grâce à la mesure des flux, l'intensité des échanges et parfois les points de passage empruntés. Cette relégation aux marges du commerce international pose cependant problème aux tenants d'approches scientifiques attachées à mettre en lumière les porosités multiples entre formel et informel, car les opérateurs des routes commerciales de produits contrefaits les redessinent sans cesse afin d'éviter d'être détectés et aussi parce que les données manquent pour avoir une quantification d'ensemble.

Entre ces deux approches méthodologiques, celle, qualitative, de l'étude des circuits d'une marchandise et celle, quantitative, de la contrefaçon, notre objectif dans le présent article est de nous attacher aux indices matériels à partir desquels une approche socio-spatiale des traces du lien transnational dans les lieux peut être esquissée. En complément des méthodes ethnographiques, notre objectif est de porter le regard sur la production de données semi-quantitatives qui permettraient de mesurer l'intensité et la pluralité de la dimension relationnelle de ces lieux et ainsi de mettre en œuvre les conditions d'une comparabilité entre les sites enquêtés quels que soient leur statut et leur visibilité. À défaut de pouvoir disposer d'un corpus statistique conséquent, voire de pouvoir en constituer un, permettant de préciser l'intensité de l'activité économique menée localement et celle des flux transnationaux qu'elle génère, il est en effet bien souvent difficile d'étayer ce point à partir d'éléments tangibles autres que les entretiens (Müller, Wehrhahn, 2013) ou les relevés (Bodomo, Ma, 2012). Les raisons en sont multiples: elles tiennent généralement autant aux approches 
compréhensives des auteurs qu'à la brièveté des investigations dans des mondes professionnels qui ne se prêtent pas facilement aux enquêtes académiques compte tenu des enjeux financiers et parfois aussi des situations délicates des personnes vis-à-vis des législations locales et nationales sur les déclarations de revenus autant que sur leurs conditions de séjour, lorsqu'elles sont étrangères. En revanche, passée la phase préparatoire faite de lectures, d'enquêtes menées sur d'autres sites et d'un premier contact avec le terrain, il apparaît rapidement que, dans chaque marché on peut s'attacher à un indice qui y apparaît comme particulièrement pertinent afin de donner une perspective diachronique et/ou synchronique au caractère transnational des marchés étudiés.

5 Les auteurs de cet article ont donc procédé en deux temps : dans un premier temps, exploratoire, ils ont mené des enquêtes de sociologie et de géographie urbaine en parallèle ou conjointes dans deux marchés parmi les plus importants à deux niveaux territoriaux différents. Il s'agit au niveau national du marché de gros d'El Eulma en Algérie dont les opérateurs bénéficient d'une quasi-exclusivité dans l'importation de produits exposés à Yiwu et au niveau mondial du comptoir de Yiwu en Chine, réputé être le marché d'exposition et de vente en gros de produits chinois le plus important du monde. L'un et l'autre sont en effet reliés de manière croissante depuis une vingtaine d'années. Après avoir échangé sur ces premiers résultats, les auteurs y ont ensuite procédé, entre 2009 et 2016, à des recensions systématiques de ce qui leur paraissait y représenter l'indicateur le plus pertinent de la dimension relationnelle de ce lieu. Ainsi, les locaux commerciaux et l'origine des produits vendus à El Eulma nous renseignent plus précisément sur les types de produits et les sources d'approvisionnement des grossistes. En complément, à l'autre bout de la chaîne commerciale, les restaurants arabes et musulmans à Yiwu sont des indicateurs de l'origine géographique des grossistes autant que de la manière dont s'organisent et comment évoluent ces places marchandes du commerce transnational (Belguidoum, Pliez, 2012, 2015, 2016, 2018). Deux indicateurs qui, en fonction du rôle et du statut de ces places situées aux deux extrémités de la route du commerce transnational entre Chine et Algérie, permettent de suivre le dynamisme de cette forme originale de mondialisation.

\section{Le souk « Dubaï » d’El Eulma (Algérie)}

6 L'Algérie est depuis plusieurs décennies un lieu privilégié d'où peut s'observer la structuration des routes du commerce transnational. Dans ce contexte, El Eulma est incontestablement la place emblématique où s'organisent les acteurs et les réseaux liés à ce commerce. Son souk «Dubaï », également appelé Shâra' par la population locale ${ }^{4}$, s'est imposé comme la principale centralité commerciale du pays pour les biens domestiques, l'équipement de la personne et de la maison.

7 Le Souk Dubaï s'étale sur plus de 110 ha et propose un linéaire de façades sur rue de $20 \mathrm{~km}$ abritant près de 3300 locaux commerciaux spécialisés dans la vente en gros, demi-gros et de détail ${ }^{5}$. De fait, El Eulma concentre une part non négligeable des importateurs algériens, puisque sur les 34000 importateurs recensés au niveau national tout type de produits confondus, 2600 sont domiciliés à El Eulma ou Sétif, soit $8 \%$ (CNRC, 2011), et les proportions sont encore bien plus importantes si on ne considère que les importateurs de produits dont El Eulma a fait sa spécialité (Belguidoum, Pliez, 2012). 
8 En une vingtaine d'années, ce qui était un petit marché de gros et demi-gros de la droguerie et de la quincaillerie s'est imposé comme le lieu incontournable de l'approvisionnement en biens domestiques et en petit outillage industriel de toute l'Algérie. Son rayonnement touche aussi la Tunisie et, via Tamanrasset, la place marchande dessert également les pays du Sahel. Si dans un premier temps, ce sont des commerçants locaux et des environs, notamment de Sétif, qui s'y sont installés, El Eulma accueille aujourd'hui des importateurs, de plus en plus nombreux venant de tout l'Est algérien et, depuis peu, de Kabylie, de l'Algérois et du Sud (El Oued, Ghardaïa). Cette attractivité croissante atteste du poids d'El Eulma dans la distribution des marchandises à l'échelle de toute l'Algérie. La place est aujourd'hui une pièce maîtresse dans le fonctionnement de la route de la soie algérienne, et son essor fait écho aux autres grandes places à partir desquelles s'organisent les routes de ce commerce, notamment Dubaï, dont le nom a été donné à son marché, et Yiwu, en Chine.

\section{Émergence de nouvelles places marchandes et redéploiement de l'armature urbaine}

9 Rien ne prédisposait cette localité dynamique, mais périphérique du territoire national à jouer un tel rôle. L'essor d'El Eulma repose sur une interaction complexe entre différents facteurs propres à la localité et au contexte global (figure 1). Les facteurs endogènes sont nombreux ; on peut citer pêle-mêle, la tradition marchande de la place, sa position sur le grand axe Est-Ouest des Hauts plateaux, à proximité de Sétif $(27 \mathrm{~km})$ et de Constantine $(100 \mathrm{~km})$, sa pépinière de jeunes entrepreneurs audacieux stimulés par les réussites fulgurantes des pionniers, durant les années 1990. À ces facteurs, il faut rajouter un contexte particulièrement favorable, celui d'un marché en pleine expansion lié à une demande de plus en plus forte et à la libéralisation des importations au milieu de la décennie 1990, jusqu'alors le monopole de l'État.

De fait, ce ne sont pas dans les grandes villes que se développent les premières places organisant ce commerce, mais dans les périphéries urbaines, dans des villes moyennes (El Eulma, Aïn M'Lila), des petites villes ou de grosses bourgades (Aïn Fakroun, Tadjenanet, Bir El Ater). Leur concentration dans l'Est algérien s'explique en partie du fait de leur plus grande proximité des frontières tunisienne et libyenne, par où provenait l'essentiel de la marchandise avant que l'importation ne se fasse par les ports algériens. El Eulma (les biens de la maison, l'ameublement, la téléphonie mobile, la micro-informatique et la quincaillerie), Aïn M’Lila (la pièce détachée pour automobile) et Aïn Fakroun (le vêtement bon marché) sont les trois localités qui, dans leur domaine de spécialisation spécifique, se sont imposées, étendant leur réseau de distribution sur l'ensemble du territoire algérien et même au-delà par l'établissement de liens avec des commerçants des pays frontaliers. D'autres localités de l'Est algérien se sont également spécialisées dans des domaines moins visibles. C'est le cas de Belaïba et El Djezar (pièces détachées pour engins de travaux publics et véhicules lourds), petites localités situées près de Barika ou encore de Bir El Ater ${ }^{6}$ dans la wilaya ${ }^{7}$ de Tébessa, qui a été un temps une des plaques tournantes des produits en provenance de Libye (Belguidoum, 2015). 
Figure 1. Les places marchandes du commerce transnational dans l'Est algérien

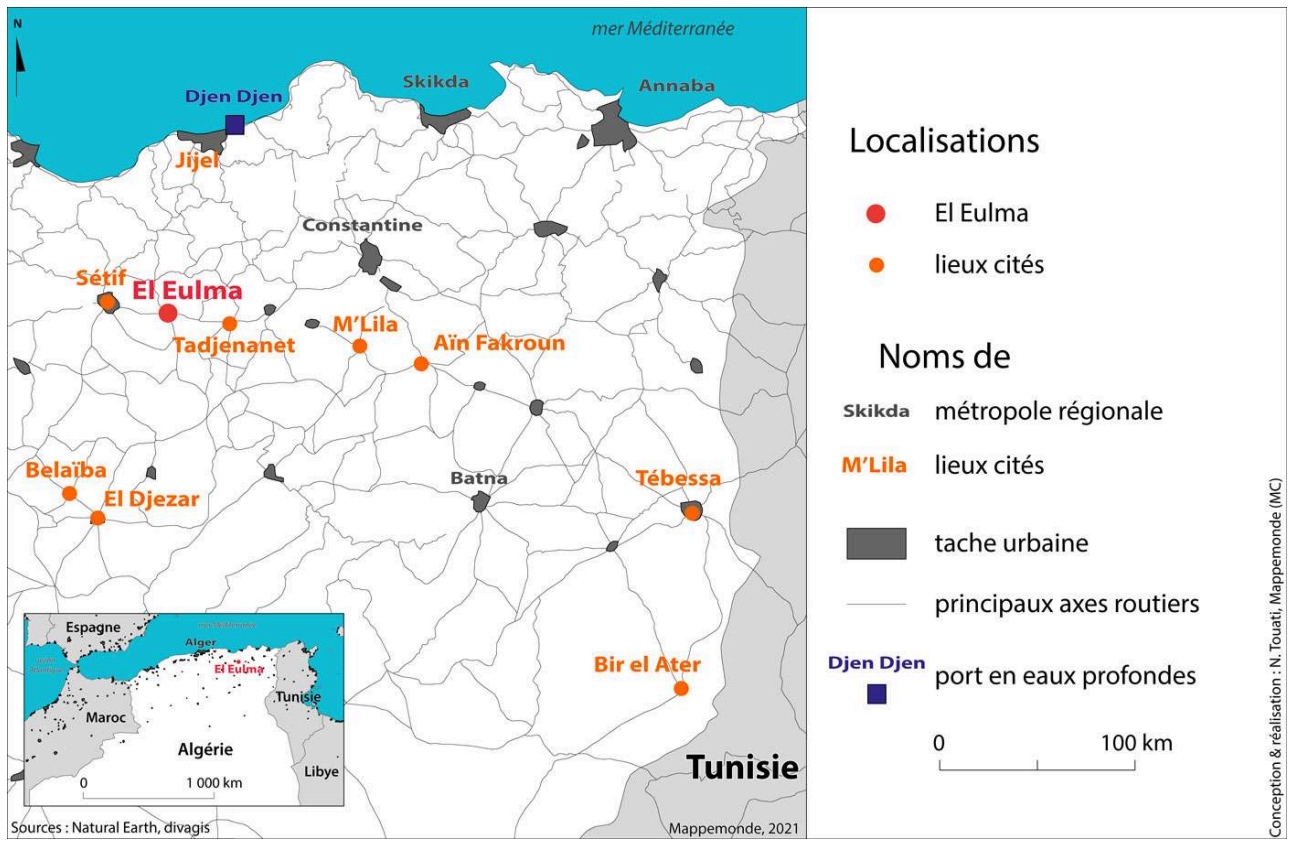

11 En s'inscrivant dans ce processus de redéploiement, El Eulma s'impose progressivement comme une place incontournable du commerce transnational en Algérie.

\section{Genèse d'un marché transnational des biens domestiques}

12 C'est à partir d'une dizaine de boutiques de quincaillerie et de droguerie, installées en 1999 en périphérie de la ville dans une petite promotion immobilière faisant face à la nouvelle gare routière, que naît ce qui deviendra l'année suivante le Souk Dubaï, appelé ainsi en référence à la provenance des marchandises. Au commerce de la quincaillerie et de la droguerie s'ajoute, entre 2000 et 2005, celui des meubles et des articles de maison, de la téléphonie mobile, de la micro-informatique, de l'électroménager. Le marché se développe rapidement en prenant possession d'un lotissement pavillonnaire créé dans son prolongement et qui change rapidement d'affectation. En 2005, un nouveau lotissement, situé sur la partie sud, est à son tour annexé au marché. Depuis 2010, la progression se poursuit, englobant au nord-ouest de la ville un périmètre de plus en plus vaste (voir figure 2). 
Figure 2. Localisation du souk Dubaï à El Eulma

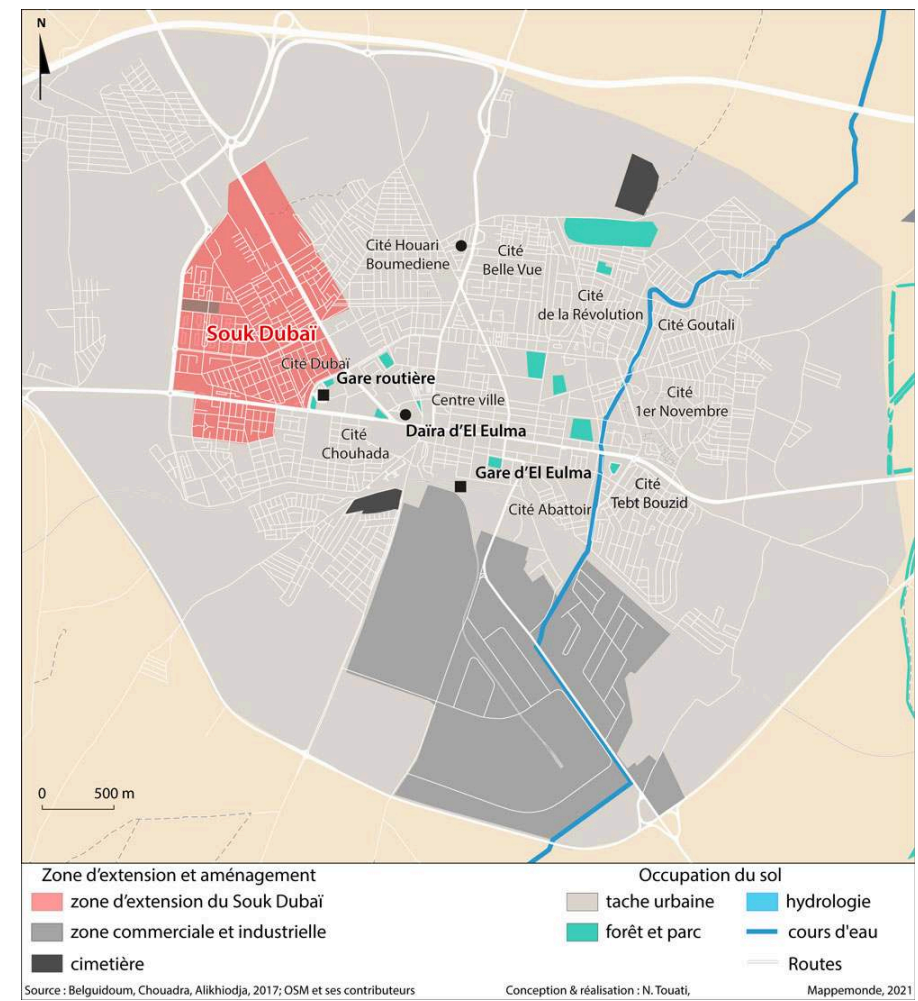

En même temps que le marché s'étend, de nouvelles activités se développent. L'habillement et les articles de sport, la lingerie et la maroquinerie font leur apparition à partir des années 2010. En avril 2015', on recense 3249 locaux de commerce, dont 2 337 sont en activité, les autres servant de dépôt ou s'apprêtant à ouvrir. Ces commerces pratiquent la vente en gros et demi-gros et sont très majoritairement spécialisés dans l'équipement domestique (biens de la personne et de la maison). Le petit outillage industriel (quincaillerie, électricité et petites machines) et l'équipement commercial (enseignes, équipements de magasin et de restaurant) ont également une présence notable.

Figure 3. Répartition des commerces par grand type

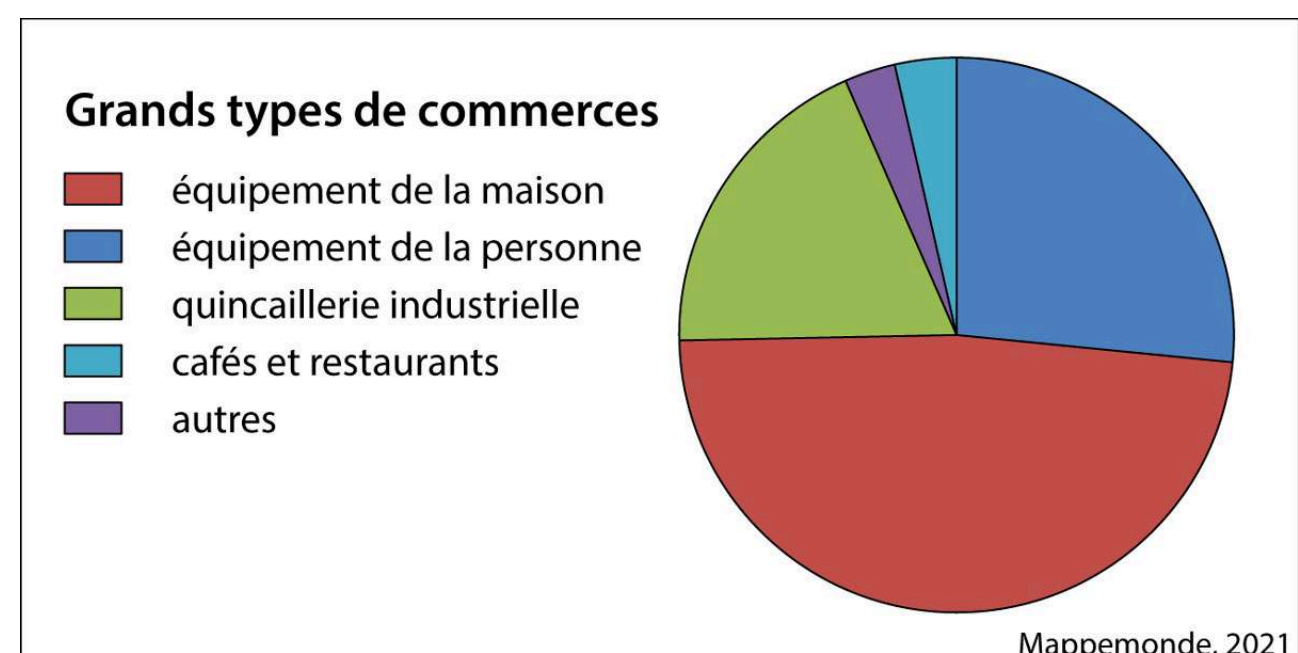

Source : Enquête systématique menée en avril 2015, par Belguidoum et al., 2017 

, les activités se déploient au sein de cinq grands secteurs, correspondant aux différentes phases d'expansion du marché (voir figure 2). Un sixième secteur s'est constitué à la fin $2015^{\circ}$, à proximité de l'autoroute Est-Ouest et de la jonction avec la future autoroute qui reliera El Eulma au tout nouveau port en eau profonde de Djen Djen (Jijel) (voir figure 4).

Figure 4. Les différents secteurs du souk Dubaï d'El Eulma

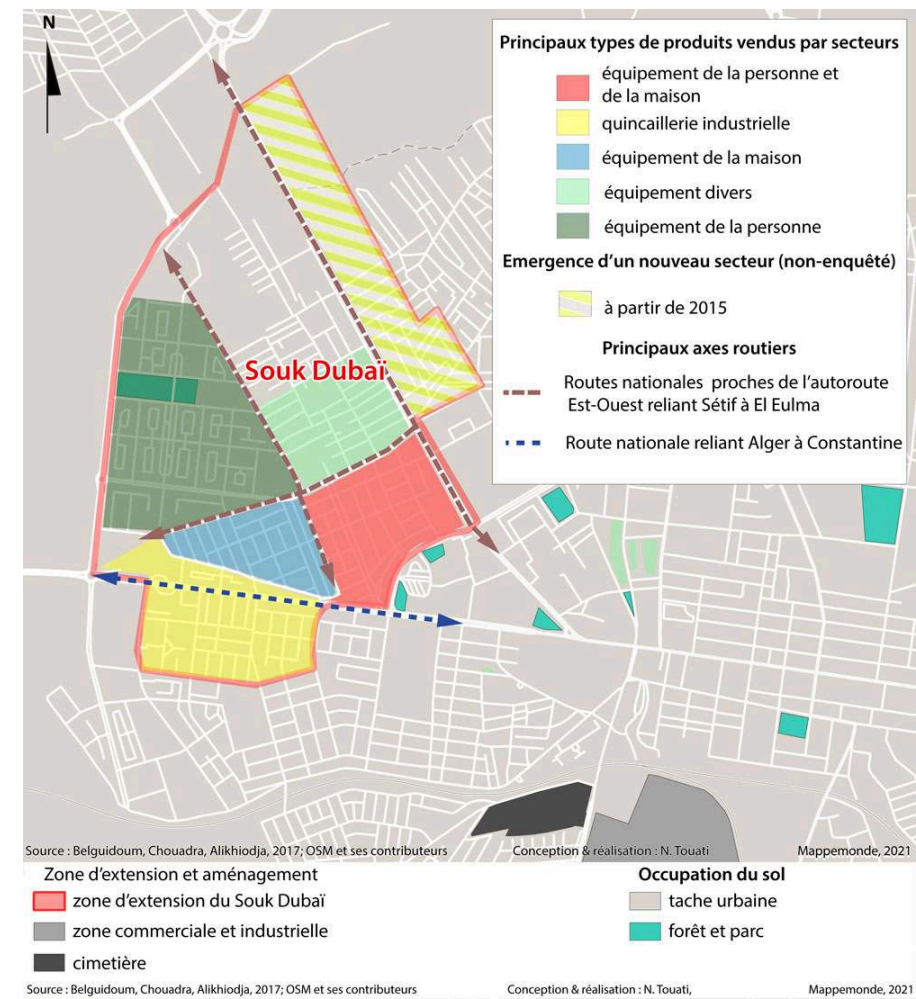

De taille inégale, chaque secteur regroupe tendanciellement des activités de même type de produit. Ainsi, à titre d'exemple, 80 \% des locaux spécialisés dans le vêtement, 93,5\% des magasins de jouets, $98 \%$ de la maroquinerie, 94,6 \% de l'électroménager et $82 \%$ de la droguerie sont concentrés dans le secteur 1. L'ameublement se répartit entre les secteurs $3(43,5 \%)$ et $4(45,9 \%)$ (voir la figure 5 et le tableau 1). 
Par ce jeu de regroupement et de spécialisation des secteurs, des îlots et des rues, les différentes activités sont facilement localisables dans le marché. Cette visibilité de l'activité est encore plus manifeste à l'échelle de la rue et du type précis de produits.

Figure 5. Répartition par secteur des grands types de commerce

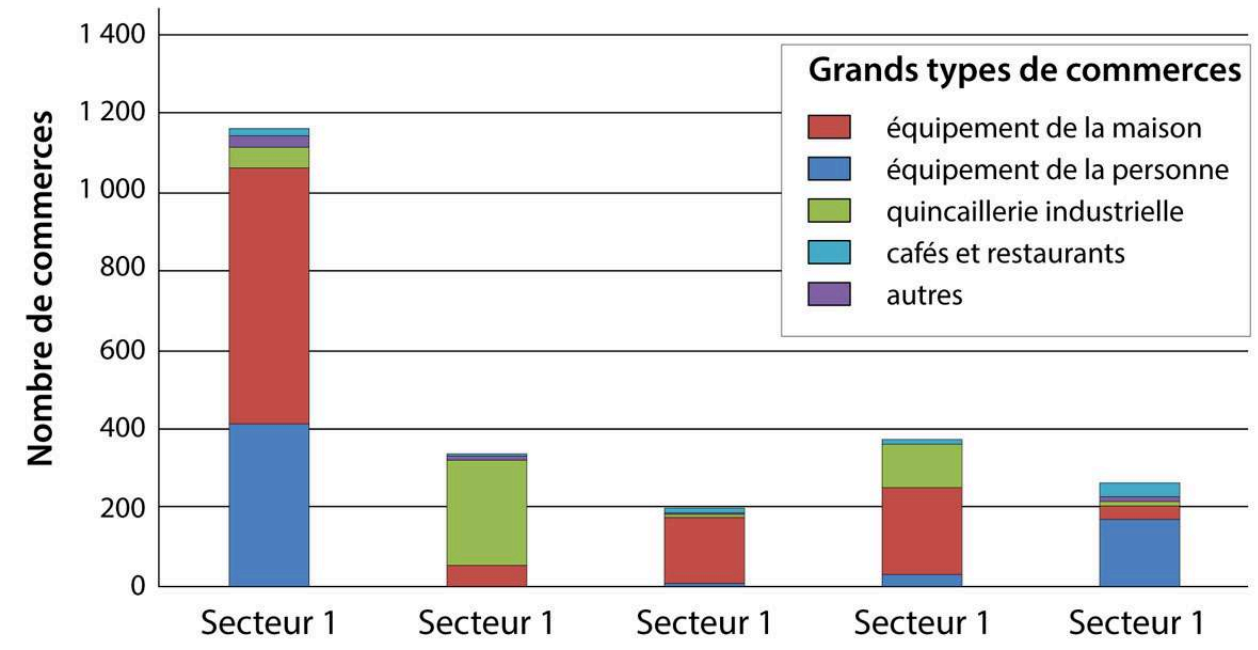

Mappemonde, 2021

Source : Belguidoum et al., 2017

Cette connexion d'El Eulma avec les grandes places du marché transnational se retrouve à l'échelle des branches de produits qui sont localisées dans des secteurs précis du marché. De fait, chaque secteur est connecté de manière particulière à une place globale du commerce transnational (tableau 1).

Tableau 1. Répartition par grand secteur et principaux lieux d'approvisionnement des commerces

\begin{tabular}{|l|l|l|}
\hline Type de commerce & Principale localisation & Lieux d'approvisionnement \\
\hline Habillement & $80 \%$ secteur 1 & Guangzhou, Yiwu, Shishi (Chine), Turquie \\
\hline Lingerie & $98,6 \%$ secteur 1 & Yiwu (Chine) \\
\hline Articles de sport & $58 \%$ secteur 4 & Guangzhou, Yiwu (Chine) \\
\hline Cosmétique & $82 \%$ secteur 1 & Yiwu (Chine) \\
\hline Maroquinerie & $98,6 \%$ secteur 1 & Guangzhou, Yiwu (Chine) \\
\hline Chaussure & $96 \%$ secteur 5 & Guangzhou, Yiwu (Chine) \\
\hline Jouets & $93,5 \%$ secteur 1 & Yiwu (Chine) \\
\hline Meubles & $\begin{array}{l}43,5 \% \text { secteur } 3 \\
\text { secteurs } 4\end{array}$ & Yiwu (Chine), Turquie, Égypte \\
\hline Textile et linge de maison & Tous les secteurs & Yiwu (Chine) \\
\hline
\end{tabular}




\begin{tabular}{|l|l|l|}
\hline $\begin{array}{l}\text { Articles de maison et } \\
\text { luminaire }\end{array}$ & $76 \%$ secteur 1 & Yiwu (Chine) \\
\hline Droguerie & $82 \%$ secteur 1 & Yiwu (Chine) \\
\hline Electro-ménager & $94,6 \%$ secteur 1 & $\begin{array}{l}\text { Shenzhen (Chine), Bordj Bou Arredj, Sétif } \\
\text { (Algérie) }\end{array}$ \\
\hline $\begin{array}{l}\text { Informatique } \\
\text { téléphonie }\end{array}$ & $71,5 \%$ secteur 1 & Shenzhen (Chine) \\
\hline Quincaillerie - électricité & $92 \%$ secteur 2 & Guangzhou, Yiwu (Chine) \\
\hline Electronique & $86 \%$ secteur 1 & Shenzhen (Chine) \\
\hline Machines industrielles & $93,7 \%$ secteur 2 & Guangzhou, Shenzhen (Chine) \\
\hline Équipement commercial & $90 \%$ secteur 2 & Yiwu (Chine) \\
\hline Accessoire automobile & $86,2 \%$ secteur 4 & Yiwu (Chine) \\
\hline
\end{tabular}

Source : Belguidoum et al., 2017

20 Ces principaux lieux d'approvisionnement sont susceptibles à tout moment d'adaptation, de modification en fonction des aléas des marchés, des contraintes régionales diverses - la Syrie par exemple qui, durant les premières années 2000, était un lieu d'approvisionnement important pour les robes et vêtements de fêtes, est une source désormais fermée du fait de la guerre -, des évolutions de la réglementation douanière ou de la découverte de nouvelles sources. Par ailleurs, les sources européennes d'approvisionnement existent (France, Espagne, Allemagne et Italie), mais concernent plus les produits de marques (essentiellement l'électronique et l'électroménager) ou certaines niches d'opportunité (articles de maison, droguerie, quincaillerie, petite électronique).

\section{Les échelles de la place : des réseaux multiples et diversifiés}

Brassant quotidiennement des tonnes de marchandises et accueillant une multitude de visiteurs, la place combine désormais les fonctions de marché de gros et de redistribution sur le marché national autant que celle de marché de demi-gros et de vente au détail. Son aire de distribution s'étend sur plusieurs échelles, grâce à une dizaine d'entreprises de transport spécialisées dans l'acheminement et la distribution des marchandises, disposant d'une flotte de semi-remorques leur permettant de desservir toutes les localités d'Algérie, jusqu'au grand Sud.

Le premier niveau concerne l'acheminement de la marchandise. Les commerçants d'El Eulma, eux-mêmes importateurs ou seulement grossistes, sont en relation permanente avec leurs fournisseurs à l'étranger, notamment par le biais des transitaires installés en Chine (Belguidoum, Pliez, 2010, 2015). Phénomène nouveau, depuis 2016, des agents d'entreprises chinoises, de plus en plus nombreux, viennent directement démarcher les commerçants de la place. On rencontre ainsi dans les restaurants de la ville d'anciens 
traducteurs chinois qui travaillaient pour des transitaires algériens installés à Yiwu, traitant directement avec les importateurs locaux. Acheminée et entreposée à El Eulma, ou dans des locaux ouverts dans d'autres localités (Alger, Sétif), la marchandise est rachetée par des commerçants locaux ou venant de toute l'Algérie. Elle peut changer plusieurs fois de mains avant d'être écoulée au détail. Le volume et la rapidité de la circulation des marchandises sont les principes essentiels de fonctionnement du marché.

Le deuxième niveau de la distribution concerne les marchés régionaux hebdomadaires du gros et demi-gros. Une autre catégorie de marchands de la localité, associés aux importateurs, ou ayant racheté en seconde main, sillonne les grands marchés régionaux : Sidi Khettab à l'Ouest, Aïn El Hadjel au centre du pays et Tadjenanet pour l'Est. Les livraisons se font aussi directement auprès des commerçants de demi-gros, jusqu'à Medina J'dida, la principale place de revente de ce type de produit à Oran (Boudinar, Belguidoum, 2015 ; Bouhali, 2015).

24 Le troisième niveau correspond au commerce de détail et de demi-gros qui est assuré par les centaines de boutiques de la localité. Attirant une clientèle de plus en plus nombreuse, notamment en fin de semaine et la veille des fêtes, le commerce de détail prend une ampleur réelle, dans un contexte urbain qui s'adapte à l'accueil de ce public extérieur. L'hôtellerie, les restaurants et les fastfoods se développent et accompagnent l'attractivité croissante de la place.

Depuis plusieurs années, la place connaît un afflux d'une clientèle tunisienne de plus en plus nombreuse venant par autocar. Profitant du pouvoir d'achat que confère le dinar tunisien, un commerce à la valise s'est instauré à partir d'El Eulma. Ce tourisme commercial rencontre un véritable engouement et touche aussi la ville voisine de Sétif, grâce à son hôtellerie et son mall flambant neuf, et celle d'Aïn Fakroun, spécialisée dans l'importation du vêtement bon marché, distante d'une centaine de kilomètres.

Ces trois niveaux qui structurent la distribution sur le marché algérien s'articulent avec un quatrième, à l'échelle mondiale cette fois-ci : les sources d'approvisionnement en marchandises vendues au Souk Dubaï.

En effet, un des facteurs essentiels dans la réussite d'El Eulma est le rôle joué par les premiers commerçants locaux dans la recherche incessante de nouvelles sources d'approvisionnement. L'organisation d'un tel commerce suppose des points d'appui dans les pays où se font les achats. Le rôle des pionniers est donc essentiel. S'installant d'abord à Istanbul, les commerçants d'El Eulma prennent pied à Dubaï au milieu des années 1990 avant de poursuivre jusqu'à Hongkong, puis Yiwu en Chine. À la fin des années 1990, sur les trois grands bureaux d'Algériens de Dubaï, le plus actif était tenu par des Eulmis ${ }^{10}$. Figures pionnières, ce sont aussi ces mêmes agents qui continuent ensuite en Chine, ou qui en ouvrent la voie, et installent les premiers bureaux à Hongkong en 1999, puis à Guangzhou et à Yiwu à partir de 2001. En 2016, 12 des 50 bureaux algériens recensés à Yiwu sont tenus par des commerçants originaires d'El Eulma $^{11}$. Ces derniers sont désormais tout aussi nombreux à être installés entre Guangzhou et Shenzhen. 


\section{L'« Exotic Street » de Yiwu} al de Yiwu (Cui, Chignier-Riboulon, 2017 · Rui, 2018), il apparait que cette successstory, plus généralement annoncée par les pouvoirs publics que les phases de tassement de l'activité commerciale, découle pour beaucoup de la fréquentation initiale de Yiwu par des grossistes chinois et des importateurs commerçants depuis les années 2000.

Figure 6. Localisation de Yiwu en Chine
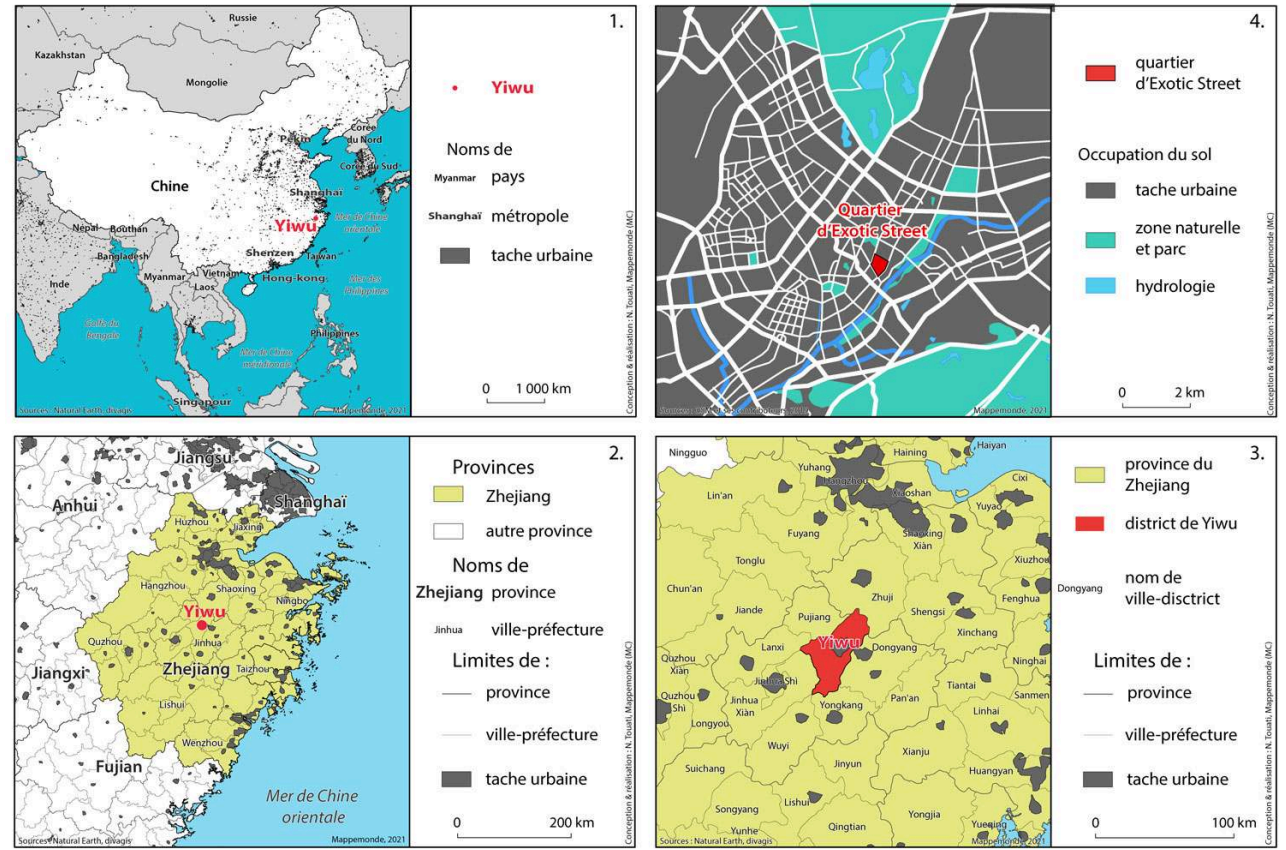

31 La genèse de Yiwu est en effet celle d'un marché régional qui est devenu national puis international entre les années 1990 et 2000 (Pliez, 2010). Lorsque la Chine adhère à l'Organisation mondiale du Commerce en 2001, Yiwu s'équipe alors d'un nouveau grand hall d'exposition des produits fabriqués dans des petites et moyennes unités de productions industrielles regroupées en clusters, l'ITC (International Trade City), à la 
signalétique en chinois, mais aussi en anglais, en arabe et en cyrillique, communément appelé Futian. Composé d'un unique bâtiment lors de son inauguration, il en compte désormais cinq dans lesquels, 364 jours par an, de $9 \mathrm{~h}$ à $17 \mathrm{~h}$, déambulent des commerçants venus du monde entier.

Ce marché est le plus visible, le plus connu, mais pour autant pas le seul dans la ville. Sa construction annonçait l'objectif de Yiwu de créer une aire de chalandise globale. Mais avant que les grossistes du monde entier ne le fréquentent, les communautés musulmanes chinoises, ouïghours du Xinjiang et Hui du Ningxia, connaisseuses des langues turcophones et parfois de l'arabe, ont donné naissance au quartier appelé alabo fan dien (« restaurant arabe » en chinois) ou El Maedah (« la table » en arabe). Le noyau de ce quartier était alors un îlot urbain de quelques rues ( $35 \mathrm{ha}$ au total), qui a été formé en plein centre de la ville pour devenir le lieu de rencontre de commerçants transnationaux.

Les musulmans chinois qui bénéficiaient, avec la chute de l'URSS, d'un débouché commercial avec l'Asie centrale ex-soviétique (Thorez, 2008) ont rapidement été rejoints par les négociants arabes qui fréquentaient les places marchandes du Golfe arabo-persique ou d'Asie du Sud-Est. Tous partageaient une pratique commune, celle de ne passer qu'un à trois jours dans la ville et de chercher des restaurants proposant de la nourriture hallal. Des boutiques exposant des produits des usines de la région y étaient concentrées; les premiers hôtels et restaurants ont ouvert autour de ces halls d'exposition. Les commerçants arpentaient quelques dizaines de rues spécialisées sur un créneau particulier de produits (décoration, informatique, papeterie...) ou quelques marchés textiles tels que celui de Binwang.

\section{Autour des marchés, des restaurants}

Le premier restaurant ouvert par un étranger dans le quartier, dès la fin des années 1990, est égyptien, nommé $E l$ Maedah ; d'autres suivront, généralement créés en association entre un ressortissant chinois, qui est le propriétaire officiel et un partenaire étranger, qui investit et gère (ou fait gérer) le lieu. Les recensions effectuées sur place démontrent que ce quartier est loin d'être statique.

Entre 2009 et $2018^{13}$, le nombre total de restaurants varie de manière conséquente, de 33 en 2009 à 40 en 2018, avec une baisse significative entre 2009 et 2012, sans doute à mettre en lien avec le tassement des importations en 2008, 2009 et 2011 (Cui, ChignierRiboulon, 2017). Ces baisses sont plus explicites par aires régionales (voir tableau 2 et figure 7). Ainsi, si le Maghreb est toujours faiblement présent, le Proche-Orient arabe occupe une place prépondérante bien qu'en baisse constante (de 20 restaurants en 2009 à 13 en 2018). A contrario, le Moyen-Orient non arabe et l'Asie centrale sont de plus en plus présents, de même que la Chine.

Tableau 2. Les restaurants du quartier Exotic Street (Yiwu, Chine)

\begin{tabular}{|l|l|l|l|l|}
\hline & 2009 & 2012 & 2015 & 2018 \\
\hline Total & 33 & 28 & 37 & 40 \\
\hline dont & & & & \\
\hline
\end{tabular}




\begin{tabular}{|c|c|c|c|c|}
\hline Maghreb & 1 & $\mathbf{0}$ & $\mathbf{0}$ & 2 \\
\hline Algérie & 1 & 0 & 0 & 0 \\
\hline Tunisie & 0 & 0 & 0 & 2 \\
\hline Proche-Orient & 20 & 14 & 11 & 13 \\
\hline Égypte & 8 & 5 & 2 & 2 \\
\hline Syrie & 5 & 3 & 1 & 2 \\
\hline Irak & 3 & 2 & 2 & 1 \\
\hline Jordanie & 1 & 1 & 1 & 1 \\
\hline Yémen & 3 & 3 & 5 & 5 \\
\hline Liban & 0 & 0 & 0 & 2 \\
\hline Moyen-Orient non arabe & 3 & 3 & 8 & 9 \\
\hline Irak (Kurdistan) & 2 & 2 & 2 & 2 \\
\hline Turquie & 0 & 0 & 5 & 6 \\
\hline Iran (kurde) & 1 & 1 & 1 & 1 \\
\hline Asie centrale & 2 & 2 & 6 & 5 \\
\hline Ouzbékistan & 0 & 0 & 1 & 1 \\
\hline Pakistan & 2 & 2 & 2 & 2 \\
\hline Afghanistan & 0 & 0 & 3 & 2 \\
\hline Chine & 4 & 4 & 7 & 7 \\
\hline Chine (Xinjiang) & 1 & 1 & 4 & 5 \\
\hline Chine (Gansu) & 1 & 1 & 1 & 1 \\
\hline Chine (Qinghai) & 1 & 1 & 1 & 1 \\
\hline Chine (Ningxia) & 1 & 1 & 1 & 0 \\
\hline Autres & 3 & 5 & 5 & 4 \\
\hline Kurde indéterminé & 0 & 0 & 1 & 1 \\
\hline Indéterminé & 3 & 5 & 4 & 3 \\
\hline
\end{tabular}

Source : enquêtes (Belguidoum, Pliez) 
Figure 7. Les restaurants du quartier Exotic Street (Yiwu, Chine) entre 2009 et 2018, selon leur aire géographique

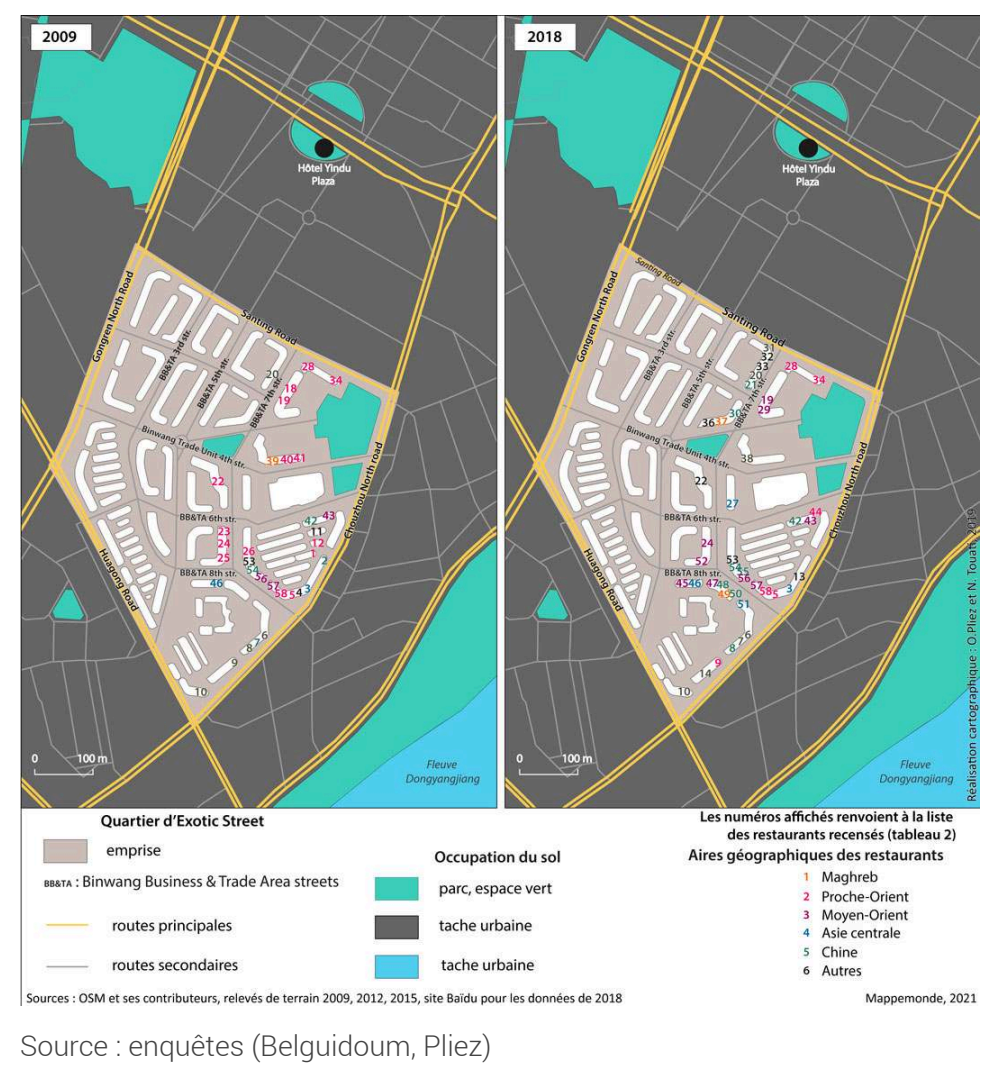

Plusieurs raisons peuvent expliquer ces différents constats. On peut spontanément penser que les conséquences des troubles qui ont secoué le Monde arabe depuis 2011 ont joué comme un accélérateur de la déprise, surtout après le tassement de l'activité économique de la fin des années 2000. Plus généralement, les liens entre les grands événements géopolitiques et l'installation dans la ville de Yiwu d'un restaurant sont multiples. Les situations de conflits attirent les grossistes du Monde arabe après le 11 septembre 2001 puis entraînent soit des situations de refuge (Irakiens en 2006, Yéménites en 2015), soit de reflux (Syriens et Égyptiens en 2011). De même, une nouvelle aire d'approvisionnement se dessine, moyen-orientale plutôt que méditerranéenne, et progressivement le quartier arabe devient un quartier musulman, majoritairement fréquenté par des non-Arabes. Plusieurs hypothèses sont alors étayées par les entretiens: dans certains cas, la maturité des réseaux ne nécessite plus la présence d'un restaurant comme pour les Algériens d'El Eulma qui se retrouvent d'abord dans un restaurant tenu par un concitoyen, le Tassili, plus tard renommé El Bahja lorsqu'il est repris par un autre Algérien avant que celui-ci ne ferme et que les grossistes ne se retrouvent dans une cafétéria chinoise. En fait, les relais de l'importation vers l'Algérie sont suffisamment anciens et bien installés en Chine, mais aussi dans d'autres places commerciales, pour que la présence d'un restaurant comme lieu d'échange d'information ou de garantie des sommes engagées ne se justifie plus vraiment. Dans d'autres cas, la succession des crises politiques et géopolitiques suscite des installations pionnières permettant à quelques-uns de ses membres d'occuper une position centrale dans des réseaux transnationaux qui dépassent les frontières d'une communauté nationale (Égypte, Syrie) jusqu'à ce qu'une autre crise provoque le 
délitement du réseau. On voit alors poindre des sorties de crise qui se traduisent par une présence croissante d'acheteurs venant de marchés de consommation émergents (Afghanistan).

\section{Conclusion}

37 À travers ces grands traits de l'organisation des circuits commerciaux, on devine une réelle stratégie qui fait la force de la place marchande d'El Eulma. Les différents niveaux scalaires d'intervention s'articulent et attestent de l'esprit d'entreprise et de conquête de marché qui animent ces nouveaux entrepreneurs, en quête permanente de filières d'approvisionnement (aujourd'hui essentiellement la Chine), de diversification des partenaires commerciaux, de pénétration des principales places régionales de vente et de fidélisation des clients les plus importants.

Cette stratégie touche aussi l'organisation urbaine, et les retombées sur l'immobilier et les pratiques constructives sont nombreuses, et pas seulement à El Eulma. La réussite des commerçants se vérifie en effet aussi par leur investissement dans la création de centres commerciaux dans les grandes villes algériennes, notamment à Sétif, Constantine, Alger (quartier d'El Hamiz) et Oran (Bouhali, 2015). Le comptage des boutiques et des types d'articles qui y sont vendus ainsi que le traçage des sources d'approvisionnement sont déterminants pour la mise en lumière de ces jeux d'échelles qui, de la boutique au monde, montrent comment une place marchande transnationale se construit et évolue.

39 S'intéresser à un comptoir comme celui de Yiwu, c'est occulter (volontairement) le fait que des quartiers du même type se sont formés dans la ville à l'initiative d'autres communautés marchandes (voir par exemple Ibañez-Tirado, 2018) autant que le fait que tous les restaurants arabes et/ou musulmans de Yiwu ne se situent pas dans ce quartier. Ils y sont néanmoins en nombre prépondérant et avec une ancienneté qui permet de considérer ce quartier comme un "baromètre » des relations établies entre Yiwu et les communautés musulmanes du monde entier, permettant d'affiner les étapes de l'évolution du quartier autant que son investissement à un moment, plus ou moins durable, par un groupe plutôt qu'un autre. Il s'agit ainsi d'éviter le risque d'essentialiser la trace spatiale d'un réseau transnational en ne surévaluant pas son ethnicité ou sa dimension confessionnelle, tout en notant le besoin sans cesse renouvelé d'un ancrage spécifique (de la part) de communautés venant d'horizons très variés qui se retrouvent ici selon leur plus petit dénominateur commun.

Ainsi, considérées séparément, ces deux places marchandes constituent deux cas d'étude qui posent de nombreuses questions sur la formation des marchés transnationaux dans des contextes géographiques distincts. Il s'agit alors de privilégier l'étude située à un niveau local de la mondialisation. Considérés comme faisant partie de dispositifs relationnels de niveau mondial, El Eulma comme Yiwu nous permettent de saisir en quoi chaque marché devient un lieu qui fait écho à un autre dans un système relationnel dont l'approche reste à affiner, notamment par l'introduction de méthodes mixtes d'enquêtes. Reste alors à déterminer si cet indice peut être transformé en indicateur puis être transposé dans l'étude d'un autre site. Nos visites de multiples marchés nous conduisent à répondre par l'affirmative. 
41 L'étude de ces deux places montre que la compréhension de la mondialisation discrète passe par l'échelle des lieux plus que par celle des seuls États, et que l'évidente intégration de ces lieux aux échanges globaux passe par une modalité particulière qui est celle du transnational. Celui-ci peut y être appréhendé selon des indicateurs qui, à défaut d'être des " comparables ", n'en donnent pas moins la possibilité de comprendre ce qui fait la spécificité de chaque lieu tout autant que la manière dont ce dernier est partie prenante d'un système d'échange global.

\section{BIBLIOGRAPHIE}

BELGUiDOUM S., PLIEZ O. (2018). “Building a 'Silk Road': A view from Below of the Trans-regional Links. Between Algeria and China”. In WIPPEL S., FISCHER-TAHIR A. (dir.), Jenseits etablierter MetaGeographien. Der Nahe Osten und Nordafrika in transregionaler Perspektive, Baden Baden : Nomos, coll. « Nahoststudien. Middle Eastern Studies », p. 231-246. ISBN 978-3-8487-4416-9. En ligne : https:// hal-univ-tlse2.archives-ouvertes.fr/hal-01947273/document BELGuidoum S., PLIEZ O. (2016). "Yiwu: The Creation of a Global Market Town in China". Articulo. Journal of Urban Research, $\mathrm{n}^{\circ}$ 12. En ligne : https://articulo.revues.org/2863

BELGUIDOUM S., PLIEZ O. (2015). «Pratiques transnationales dans un comptoir de "la Route de la soie" : Algériens et Égyptiens à Yiwu (Chine) ». Les Cahiers d'EMAM, nº 26. En ligne : http:// journals.openedition.org/emam/927

BELGUIDOUM S., PLIEZ O. (2012). « Construire une route de la soie entre l'Algérie et la Chine ». Diaspora, $n^{\circ} 20$, p. 115-130. En ligne : https://journals.openedition.org/diasporas/3061

BELGUidoum S., CHOUADRA S., ALIKHODJA N. (2017). « El Eulma, anatomie d'une place emblématique du commerce transnational ». Carnets de l'IREMAM. En ligne : http://iremam.hypotheses.org/6456 BELGUIDOUM S. (2015). « Le dynamisme des nouvelles places marchandes de l'Est algérien: reconfiguration urbaine et nouvelles donnes sociales ». In PERALVA A. et VERA DA SILVA T., Ilegalismos na globalizaçao, migraçoes, trabalho, mercados, Rio de Janeiro : Editora UFRJ, p. 271-287.

BERTONCELLO B., BREDELOUP S., PLIEZ O. (2009). « Hongkong, Guangzhou, Yiwu : de nouveaux comptoirs africains en Chine ». Critique internationale, 3/2009, nº 44, p. 105-121. En ligne : https:// www.cairn.info/revue-critique-internationale-2009-3-page-105.htm BoDomo A.B., MA E. (2012). "We are what we eat: Food in the Process of Community Formation and Identity Shaping among African Traders in Guangzhou and Yiwu". African Diaspora, vol. 5, $\mathrm{n}^{\circ} 1$, p. 3-26.

BOUDINAR I., BELGUIDOUM S. (2015). « Dynamique marchande et renouveau urbain à Oran. Médina J'dida et Choupot deux quartiers du commerce transnational ». Les Cahiers d'Emam. Études sur le monde arabe et la Méditerranée, $\mathrm{n}^{\circ}$ 26/2015. En ligne : https://journals.openedition.org/emam/1027 BouHALi A. (2015). « Médina J'dida (Oran), un quartier-marché sur les routes algériennes du commerce transnational ». Les Cahiers d'EMAM, nº 26-2015. En ligne : http:// journals.openedition.org/emam/889 
CENTRE NATIONAL DU REGISTRE DU COMMERCE (2011). « Les créations d'entreprises en Algérie.

Statistiques $2010 »$. Alger.

CHOPLIN A., PLIEZ O. (2018). La mondialisation des pauvres. Loin de Wall Street et de Davos. Paris : Seuil, coll. « La République des idées », 128 p.

соок I. (2004). "Follow the Thing: Papaya". Antipode, vol. 36, nº 4, p. 642-664.

CUI C., CHIGNIER-RIBOULON F. (2017). « Yiwu : la formation d'un cluster chinois ». Revue d'économie industrielle, $\mathrm{n}^{\circ}$ 159, p. 139-158. En ligne : https://journals.openedition.org/rei/6624

IBAÑEZ-TIRADO D. (2018). "Hierarchies of Trade in Yiwu and Dushanbe: The case of an Uzbek

Merchant Family from Tajikistan”. History and Anthropology, vol. 29, suppl. 1, p. 31-47. En ligne : https://doi.org/10.1080/02757206.2018.1506776

KNOWLES C. (2014). Flip-Flop: A Journey Through Globalisation's Backroads. Londres : Pluto Press, 232 p.

LI R., WANG Q., CHEONG KC. (2016). “From obscurity to global prominence. Yiwu's emergence as an international trade hub". Cities, vol. 53, p. 8-17.

MARSDEN M., SKVIRSKAJA V. (2018). "Merchant identities, trading nodes, and globalization". History and Anthropology, 29:sup1, p. 1-13, DOI : 10.1080/02757206.2018.1497622. En ligne : https:// doi.org/10.1080/02757206.2018.1497622

MILHAUD S. (2017). « Les petites villes au centre d'une recomposition des territoires chinois. L'exemple de la province du Zhejiang ». L'Espace géographique, t. 46, nº 4, 2017, p. 311-328.

MÜLLER A., WEHRHAHN R. (2013). “Transnational business networks of African intermediaries in China: Practices of networking and the role of experiential knowledge". Die Erde, vol. 144, $\mathrm{n}^{\circ} 1$, p. 82-97.

OECD/EUIPO (2017). Mapping the Real Routes of Trade in Fake Goods. Paris: Éditions OCDE, 160 p. PERALDI M. (2007). « Aventuriers du nouveau capitalisme marchand. Essai d'anthropologie de l'éthique mercantile ». In ADELKHAH F., BAYART J.-F. (dir.), Voyages du développement. Émigration, commerce, exil. Paris : Éditions Karthala, p. 73-113. ISBN 978-2-84586-940-0

PLIEZ O. (2010). « Toutes les routes de la soie mènent à Yiwu (Chine) : Entrepreneurs et migrants musulmans dans un comptoir économique chinois ». L'Espace géographique, vol. 39, $\mathrm{n}^{\circ} 2$, p. 132-145. En ligne : https://www.cairn.info/revue-espace-geographique-2010-2-page-132.htm PORTES A. (1997). Globalization from Below: The Rise of Transnational Communities. WPTC-98-01. Université de Princeton. En ligne : http://maxweber.hunter.cuny.edu/eres/docs/eres/ SOC217_PIMENTEL/portes.pdf RIVOLI P. (2007). Les Aventures d'un tee-shirt dans l'économie globalisée. Paris : Fayard, 358 p. ISBN 2-213-63221-6.

RUI H. (2018). "Yiwu : Historical transformation and contributing factors". History and Anthropology, vol. 29, suppl. 1, p. 14-30. En ligne : https://doi.org/10.1080/02757206.2018.1516654 TARRIUS A. (2002). La mondialisation par le bas : Les nouveaux nomades de l'économie souterraine. Paris : Balland, 168 p. ISBN 2-7158-1426-7

THOREZ J. (2008). «Bazars et routes commerciales en Asie centrale. Transformation postsoviétique et "mondialisation par le bas ». Revue européenne des migrations internationales, vol. 24, $\mathrm{n}^{\circ}$ 3. En ligne : http://journals.openedition.org/remi/4844 
SIMPFENDORFER B. (2009). The New Silk Road. How a Rising Arab World is Turning Away from the West and Rediscovering China. Basingstoke : Palgrave Macmillan, 208 p. ISBN 978-0-230-28485-2.

VICENTE J. (2016). Économie des clusters. Paris : La Découverte, coll. « Repères », 128 p.

ISBN 978-2-707-18595-2.

\section{NOTES}

1. C'est d'ailleurs un banquier qui a mené la première enquête remarquée sur ces réseaux émergents : Simpfendorfer (2009).

2. La formule de Max Weber (1958) est reprise par Michel Peraldi (2007).

3. «The idea of 'globalization from below' that encompasses the small-scale operations, informal economy, ethnic and diasporic networks and international migrants » in Marsden, Skvirskaja (2018).

4. En arabe, artère principale, avenue, boulevard, rue.

5. Enquête réalisée en avril 2015. Depuis le marché poursuit son expansion. Belguidoum et al. (2017).

6. À propos des nuances d'écriture : sur le site du ministère algérien des collectivités, on écrit Bir El Ater alors que dans le langage courant on dit Bir Ater (le El est avalé).

7. Wilaya : circonscription administrative correspondant au département.

8. Enquête systématique menée en avril 2015 portant sur la totalité des locaux commerciaux du souk Dubaï (Belguidoum et al., 2017)

9. Le secteur 6 s'étant constitué à la fin de l'année 2015, il ne figure pas dans notre enquête.

10. Eulmis : habitants d'El Eulma.

11. Enquête effectuée à Yiwu en septembre 2016.

12. «Regroupement localisé d'organisations de différentes natures (...) tourné vers un marché, une industrie ou un domaine technologique particulier » (Vicente, 2016).

13. Nous avons effectué des recensions à Yiwu en 2009, 2012 et 2015. Celles effectuées en 2006 ne sont pas suffisamment complètes pour être intégrées. La date de 2018 correspond aux restaurants ouverts entre 2016 et 2018 recensées sur le site chinois baidu: http:// www.go007.com/ditu/canting_yiwu/page consultée le 26 avril 2019.

\section{RÉSUMÉS}

Cet article propose une approche socio-spatiale du commerce transnational en s'intéressant à deux places situées aux deux extrémités de la route transnationale entre l'Algérie et la Chine : El Eulma et Yiwu. Ces deux places marchandes, situées dans des contextes géographiques distincts, sont reliées entre elles par des dynamiques discrètes et efficaces et sont révélatrices de la manière dont fonctionnent et évoluent des marchés transnationaux. Considérés comme un tout, El Eulma comme Yiwu permettent de saisir en quoi chaque marché devient un lieu qui fait écho à un autre dans un système relationnel global. L'étude de ces deux comptoirs montre que la compréhension de la mondialisation discrète passe par l'échelle des lieux, plus que celle des seuls États. L'article repose sur une série d'enquêtes de sociologie et de géographie urbaine menées conjointement ou séparément entre 2009 et 2016. 
This article proposes a socio-spatial approach to transnational trade by focusing on two trading posts situated at either end of the transnational route between Algeria and China: El Eulma and Yiwu. These two marketplaces, located in separate geographical contexts, are interconnected by discreet and efficient dynamics and are indicative of the processes in which transnational markets operate and evolve. When considered together as a whole, El Eulma and Yiwu provide insight into the way one market echoes another within a global relational system. The study of these two marketplaces demonstrates that the understanding of Inconspicuous Globalisation involves the local scale and not solely the national one. The article is based on a series of sociological and urban geographical surveys conducted jointly or separately between 2009 and 2016.

Este artículo propone una aproximación socioespacial al comercio transnacional. Para ello estudia dos puntos comerciales localizados en los extremos de la ruta entre Argelia y China: El Eulma et Yiwu. Estas dos ciudades comerciales, con entornos geográficos muy diferentes, están ligadas por discretas y eficaces dinámicas geopolíticas y socioeconómicas que reflejan con funcionan y evolucionan los mercados transnacionales. En un sistema relacional global El Eulma como Yiwu solo se pueden comprender valorando sus mercados como ecos de ambas ciudades. Con el estudio de estos dos puntos comerciales se puede afirmar que la globalización refuerza más la escala de los lugares que la de los estados. Para ello, el artículo utiliza una serie de encuestas de sociología y geografía urbana realizadas de forma conjunta o separada entre 2009 y 2016.

\section{INDEX}

Thèmes : Les routes de la soie existent déjà. Routes transnationales et places marchandes du made in China entre Asie Afrique et Europe

Mots-clés : Commerce transnational, comptoirs commerciaux, El Eulma, mondialisation, places marchandes, Yiwu

Palabras claves : Comercio transnacional, puntos comerciales, El Eulma, mundialización, lugares comercial

Keywords : El Eulma, Globalization, marketplaces, trading posts, transnational trade, Yiwu

\section{AUTEURS}

\section{SAIID BELGUIDOUM}

Sociologue, maître de conférences hors-classe à l'Université Aix-Marseille (AMU) et chercheur à l'IREMAM (CNRS, UMR 7310, Aix-en-Provence)

\section{OLIVIER PLIEZ}

Géographe, directeur de recherche CNRS au sein d'ART-Dev (UMR 5281 Acteurs Ressources et Territoires dans le Développement) 